\title{
PSYCHOSOCIAL FACTORS THAT CAN INFLUENCE THE SELF-ASSESSMENT OF FUNCTION
}

\author{
Robert J. Gatchel, Ph.D., ABPP \\ Department of Psychiatry \\ The University of Texas Southwestern Medical Center at Dallas \\ 5323 Harry Hines Blvd. \\ Dallas, TX 75390-9044
}

The writing of this paper was supported in part by Grants from the National Institutes of Health (2K02 MH1107, 2R01 DE10713, 3R01 MH46452) and from the Department of Defense (PRO 23002) 


\begin{abstract}
What has plagued the evaluation process in this area has been the level of agreement in the wide variation in the measures used to document a construct such as pain, as well as changes in that construct as reflected in the measurement of function. The present article reviews the major psychosocial barriers to assessment/recovery that have been implicated as influencing the selfassessment of function. The following are discussed: secondary gain; secondary loss; emotional distress (such as anger, anxiety and depression); psychopathology; somatization and symptom magnification; compliance and resistance; patient comprehension/mental status; and iatrogenic effects.
\end{abstract}


Through the past decade, it has become quite clear that pain-related disorders, especially when associated with work disability and/or financial benefits, result from a complex interaction of medical, psychological and social factors. Therefore, a biopsychosocial perspective must be taken when dealing with medical and rehabilitation issues (e.g., 1, 2). Obviously, while embracing this perspective, certain assessment/treatment complexities are encountered. These have been reviewed previously $(3,4)$. To summarize, there are three broad categories of measures - physical, psychological/self-report, and overt behavior/function - that all need to be considered. However, these three major measurement categories (or biopsychosocial referents) may not always display high concordance with one another when measuring a construct such as pain. Such less-than-perfect concordance among these biopsychosocial referents of a construct is not unique to the area of pain or rehabilitation medicine in general. For example, it has long been noted in the psychology literature that self-report, overt behavior, and physiological indices of behavior in general sometimes show low correlations among one another. Therefore, if one uses a self-report measure as a primary index of a construct such as pain, and compares it to the overt behavioral or physiological index for the same construct, direct overlap cannot automatically be expected. What makes this even more complex is the fact that two different self-report/psychological indices or physiological indices of the same construct may not be as highly correlated as one would desire. What has really plagued the evaluation arena in general has been the lack of agreement in the wide variation in the measures used to document a construct such as pain, as well as changes in that construct. Therefore, the literature is replete with many different measurement techniques and tests of a construct such as pain, including the measurement of function. 
With the above in mind, there are a number of important "assumption traps" that health care professionals should avoid when considering the best outcome measure to utilize. These assumption traps are listed in Table 1. It should be kept in mind that, whenever evaluating a construct such as pain or function, one cannot assume that there will be one "gold standard" measure which will be the most reliable or valid. Rather, a multidimensional approach will usually need to be employed in evaluations.

\section{INSERT TABLE 1 ABOUT HERE}

In more specifically considering psychosocial factors that can influence the selfassessment of function, some of the major psychosocial barriers to assessment/recovery that have been implicated as influencing the self-assessment of function will be discussed in greater detail.

\section{SECONDARY GAIN}

In the area of pain and function, issues of secondary gain have traditionally been viewed as major barriers to recovery, especially in patients with workers' compensation injuries.

Disability behaviors are thought to be perpetuated by the perceived financial, vocational and emotional awards that might arise from the psychosocial context of "being sick" for an extended period of time. Secondary gain associated with illness or injury was frequently and erroneously equated with conscious malingering, particularly when the potential for financial gain was apparent. However, as will be discussed, secondary gain issues need not be major barriers to recovery in patients, and can actually do a disservice to patients who may be erroneously labeled as unmotivated and resistant to treatment. As will be reviewed, secondary loss issues (such as 
financial, relationship loss and the emotional consequences of loss) are extremely important to consider in any assessment or treatment process.

What is Secondary Gain?

Sigmund Freud originally introduced the concept of secondary gain, which he described as "...interpersonal or social advantage obtained by the patient as a consequence of ...illness."

(5). This should be differentiated from primary gain, which is conceptualized as an intrapsychic phenomenon by which anxiety is reduced through an unconscious defensive operation resulting in symptoms of a physical illness. Blindness, paralysis of a limb, or chronic pain for which a medical etiology cannot be isolated are examples of symptoms of illness perceived to be mediated by primary gain. Ultimately, the diagnoses of "hysteria," a conversion disorder, or a "nonorganic" chronic pain syndrome were made in these patients.

As Leeman, Polatin, Gatchel and Kishino (6) have reviewed, the basic concepts of primary and secondary gain were originally thought of as occurring along a continuum, in which secondary gain resulted from the symptoms initially created by primary gain mechanisms. A patient's need to alleviate guilt or conflict was expressed in the physical symptoms (primary gain). A patient then was able to avoid certain activities is order to receive support from his/her environment that otherwise would not be forth-coming (secondary gain). Fishbain (7) reviewed examples of secondary gain cited in the literature. They may range from the gratification of unspoken needs and wishes (e.g., to be dependent and taken care of; to take revenge; to change family dynamics), to more material concerns (e.g., financial gain; solicitation of drugs; avoiding sex; maintaining family and relationship dominance). 


\section{$\underline{\text { Secondary Gain and Assessment/Treatment Outcomes }}$}

In recent years, secondary gain has taken on a life of its own outside the traditional psychoanalytic arena, especially in the area of pain management. Its association with an illness or injury is frequently and erroneously equated with conscious malingering, especially when the perceived scope of secondary gain is limited to financial issues (8). However, a much more enlightened view is that disability and abnormal illness behaviors will be stimulated by financial compensation according to basic principles of operant conditioning; that is to say, if patients are being paid to be sick, they will learn to continue those behaviors that reward them (9). This might lead to a simplistic explanation of cause and effect. Whatever the theoretical construct, we would assume that, once the cause is eliminated, the effect disappears. Therefore, a common clinical assumption usually made is that, once financial claims are successfully resolved, the alleged illness improves. However, researchers have not confirmed this but, rather, have found that while exaggerated self-reported physical symptoms are more extreme when financial secondary gain exists (10), favorable resolutions of financial claims do not necessarily resolve the perceived dysfunction or disability (11).

Unfortunately, many health care professionals have made the assumption that patients with financial secondary gain issues are "untreatable" or "untestable" (12). However, this assumption is seriously challenged by clinical studies demonstrating favorable treatment outcomes even in the presence of unresolved financial secondary gain claims (e.g., 13). One of the serious risks of an excessive focus on secondary gain is a premature and unjustified therapeutic discouragement, so that the clinician may rationalize a denial or lessening of care because the patient's prognosis is thought to be poor. As a consequence, surgery and 
conservative care may be restricted. None of this may ultimately be in the best interest of the patient (14).

Although a patient with financial secondary gain issues overlying clinical complaints may be frustrating to the clinician, certain realities need to be recognized. First of all, in such a patient, self-heightened report measures of pain, suffering, disability and emotional dysfunction (e.g., anxiety and depression) will probably be higher than in a patient with no secondary gain issues, and these measures may not improve very much with treatment, even though functional measures do improve. Therefore, when evaluating or treating such a patient, the focus should be on the correction of functional deficits and not necessarily on rendering the patients symptomfree (15). In fact, the clinician's orientation in such cases should be a biopsychosocial one, in which not only health issues are addressed, but also labor market conditions, local economic characteristics, work environments, educational levels, household factors, and cultural values (16). In order to most effectively manage patients with pain as a major workers' compensation issue, it is important to have an understanding of the barriers to recovery that have the potential to delay clinical improvements and case resolution. Being sensitive to these barriers leads to a better understanding of a patient's motivation to change and to comply with a particular treatment or rehabilitation program. Treatment staff, case managers and risk managers all may be more effective in their jobs and accomplish appropriate and compassionate case closure by comprehensively addressing these barriers to recovery. Invariably, disability behaviors are perpetuated by perceived financial, vocational and emotional rewards that arise from the psychosocial context of "being sick" for a long period of time. Changing these behaviors requires that these reinforcers be modified to encourage a more adaptive lifestyle. Techniques to 
accomplish this transcend conventional medical or psychological treatment. These techniques will be discussed next.

\section{Techniques for Managing Secondary Gain Issues}

When a patient has failed to respond as expected to a treatment regimen, that patient may need to be re-evaluated from a biopsychosocial perspective in order to review all of the psychosocial, financial, occupational, family, educational and cultural factors that may be influencing the expression of the pain and disability. After this re-evaluation, the clinician will need to define a medical endpoint for the patient, even in the face of the patient's strong opposition. The clinician will need to organize all available medical, psychosocial and vocational resources to implement a treatment plan that will be the final effort to resolve all pain disability issues, and to return the patient to as normal a life as possible. Everyone who is involved in the patient's disability - - family members, health care providers, insurance claims manager, attorneys - - may have to be enlisted to endorse this final rehabilitation effort in order to ensure its therapeutic success. Indeed, in order to manage secondary gain issues, an assembled treatment team must accomplish the following:

- Establish trust and rapport.

- Involve a disability case manager in the treatment process.

- Contain any financial secondary gain.

- Incorporate vocational planning in the treatment program.

- Employ a multi-modal disability management program.

Leeman and colleagues (6) discuss methods that can be used in realizing the above goals.

In summary, the management of secondary gain in pain patients requires an awareness of biopsychosocial issues applicable to the particular case. Problematic and self-defeating 
behaviors can be controlled only when an overt dialog has been established with patients for whom specific concerns are directly addressed by the treatment staff. This requires the healthcare practitioner to understand the educational, financial and occupational expectations of patients and their families, as well as the realities of the insurance system in which the injury or medical condition is being covered. Educating the patient about reasonable expectations may occur only after trust has been established. The health-care practitioner must be a resource first, and an advocate thereafter, in order to help a patient resolve secondary gain issues and effect a successful recovery and resumption of normal activities of daily living.

\section{SECONDARY LOSS ISSUES}

Although secondary gain issues discussed above may be major barriers to recovery, an equally large barrier to effective assessment/treatment of pain patients may be the extensive personal losses that can arise as secondary features of chronic pain. While the role of "secondary loss" in the well being of the chronic pain patient has not traditionally received significant attention among health care professionals, a new line of inquiry is emerging to examine its influence. These losses can infiltrate almost all domains of a patient's life, including autonomy, self-esteem, social relationships, employment, and even familial roles. Indeed, according to Fishbain (7), some of the common secondary losses associated with the sick role are listed below.

- $\quad$ Economic loss

- Loss of social relationships at work

- Loss of one's social support network

- $\quad$ Social stigma of being disabled

- Guilt over disability 
- Loss of recreational activities

- Loss of respect from family and friends.

Thus, secondary loss issues are extremely important to consider in any pain-and-function evaluation process. Losses, such as financial, relationship loss and the emotional consequences of loss, can have a "cascade" effect, leading to increased emotional distress experienced by chronic pain disability patients. Unfortunately, health care professionals have traditionally ignored such loss issues. Any comprehensive treatment approach to pain disability, especially if it is chronic, will need to address such secondary loss issues and their emotional/distress sequelae in order to maximize treatment gains. The issue of emotional distress will be discussed next.

\section{EMOTIONAL DISTRESS}

In addition to secondary gain and secondary loss, there are other psychosocial barriers to assessment/recovery, as outlined in Table 2. These others will be discussed, starting with emotional distress. Because pain is ultimately a subjective, experiential state, there are a great number of affective properties that may affect assessment. Turk and Monarch (2) have highlighted the most important of these affective factors. They are briefly reviewed below.

\section{INSERT TABLE 2 ABOUT HERE}

\section{$\underline{\text { Depression }}$}

In an early study, Romano and Turner (17) indicated that from $40 \%$ to $50 \%$ of all chronic pain patients experienced some form of depression. Usually, this depression is situational in 
nature, reflecting patients' reaction to their suffering with pain. Therefore, clinicians need to be aware of the possible effect of depression on the evaluation process.

\section{$\underline{\text { Anxiety }}$}

Anxiety is also a very prevalent concomitant of pain, especially in chronic pain. Painrelated fear and concerns about avoiding additional harm and pain is quite common. Indeed, the threat of intense pain will "capture the attention" of anyone, and is also frequently difficult to disengage from. The constant vigilance in the monitoring of pain stimulation, and the oftenmisguided belief that it may signify a progression of a disease, will result in even low intensity nociception or pain to become unbearable. As Turk and Monarch (2) have indicated, the fear of pain, which is driven by the anticipation of pain and not necessarily the sensory experience of pain, will prompt a great deal of avoidance behavior and retreat from normal functional activities. Such avoidance behavior is reinforced in short-term, because of the reduced suffering associated with nociception. However, such avoidance will eventually be quite maladaptive if it persists because it will lead to increased fear, limited activity and other consequences that will significantly contribute to prolonged disability and persistence of pain. Indeed, there have been some studies to demonstrate that fear of movement and fear of re-injury are better predictors of functional limitations than actual biomedical parameters (e.g., 18).

Anger

There has been increased attention on anger as a commonly observed affective state in patients with chronic pain (e.g., 19). Such anger may be precipitated by factors such as frustration associated with the persistence of symptoms and repeated treatment failures, anger towards employers and the workplace where the pain-related injury may have occurred, with insurance companies and the health care system in general because of the difficulty that is often 
encountered in trying to receive appropriate treatment, family members who are perceived as non-sympathetic, etc. The negative consequences of such feelings of anger have been pointed out by Kerns and colleagues (20), who suggested that internalization of feelings of anger was strongly related to measures of pain intensity, reported frequency of pain behaviors, and perceived interference with activities of daily living. Although the precise mechanism by which anger and frustration exacerbate pain are not known, the role of heightened autonomic arousal has been implicated (2). Indeed, patients who are angry at the health care system in general, for example, will likely be less motivated to totally respond to an evaluation process.

Overall, besides the above emotional factors delineated by Turk and Monarch (2), there are a number of other psychosocial factors known to influence the experience of pain. These include the following:

- Uncertainty/ambiguity on the part of patients as to what to do

- Unpredictability of how long the symptoms may last

- Expectancy/anticipation factors as to what the best treatment will be

- The meaning of the symptoms

- Environmental factors that may influence pain symptoms (e.g., inadvertent reinforcement of pain symptoms by others).

- Entitlement issues generated from a sense of feeling misunderstood, cheated and betrayed. The financial and material losses they may have sustained can add to a belief that someone (or everyone) involved in the compensation process "owes me."

The health care professional will need to address/deal with these above factors during initial contact with patients in order to reassure them and prevent unwanted contributions to pain enhancement. 


\section{PSYCHOPATHOLOGY}

The presence of some major types of psychopathology can also often affect the assessment process. The DSM-IV is the official diagnostic manual of the American Psychiatric Association (21). The DSM-IV sorts the physical, psychologic and historical components of a problem behavior into five categories or axes:

- Axis I consists of clinical syndromes, and is used to designate major thought disorders and psychiatric problems such as schizophrenia, affective disorders and substance abuse disorders.

- $\quad$ Axis II designates personality disorders (adults) and specific developmental disorders (children and adolescents). Some examples of Axis II diagnoses would be anti-social and borderline personalities.

- $\quad$ Axis III denotes the patient's medical diagnosis. The DSM-IV considers knowledge of the general physical health of the person to be a major part of the total diagnostic picture. For example, a patient with a herniated nucleus pulposus would have that diagnosis listed under Axis III. However, if a specific organic or pathophysiologic diagnosis cannot be made, the pain problem would be noted under Axis III, but this time by the patient's symptoms.

- Axis IV (psychosocial and environmental problems) enables the clinician to rate specific psychosocial events that are judged to be significant contributors to the development or exacerbation of the present disorder. For example, financial conditions and occupational status may have etiologic significance and, therefore, be considered psychosocial stressors.

- Axis V (global assessment of functioning) allows the clinician to rate the patient's overall psychological, social and occupational functioning on the global assessment of functioning (GAF) scale, which assesses mental health or illness. Ratings on this scale are made for two 
time periods, current (level of functioning at the time of evaluation) and at some other time period.

Of course, many forms of psychopathology can obviously affect the assessment process. For example, if the individual has some major thought disorder (such as a psychotic disorder), this may affect the reliability of information garnered from that patient. Likewise, an antisocial personality disorder may be associated with inaccurate or deliberate falsification of information. Therefore, such psychopathologies need to be carefully considered when deciding the credibility of the assessment process.

\section{SOMATIZATION AND SYMPTOM MAGNIFICATION}

It is important to understand that certain patients use their physical symptoms as a way of dealing with, and communicating about, their emotional lives (somatization). That is to say, in this type of symptom magnification, physical symptoms may be easier to accept as causing current unhappiness and discontent than admitting that some physiological reason is contributing to it. Only rarely is a patient consciously "faking" functional disability, although many times symptoms may be exaggerated consciously or unconsciously. Symptoms may also be magnified as a way of "saving face" and justifying continued functional disability after a certain period of dysfunction. This may, therefore, reflect conscious or unconscious illness affirmation. These processes are most apparent in the realm of self-report of pain-and-function measures. It is essential for assessment personnel to carefully delineate such issues and develop an appropriate strategy to affectively deal with them. It should also be noted that this issue of symptom magnification, because it is so important in the area of chronic pain and disability, is generating a great deal of research interest.

\section{COMPLIANCE AND RESISTANCE ISSUES}


Unlike athletes, who usually have great motivation to be assessed in order to start a treatment program, many "ordinary" pain patients may tend to be reluctant to start a program to regain function too quickly. Often, this is related to fears of reinjury, musculoskeletal inhibition, or other psychosocial barriers. Therefore, in the initial assessment process, when fear and trust are usually major issues for the patient, noncompliance or failure to cooperate should be dealt with in a supportive and educational manner. A great deal of time and effort should be put into explaining the assessment rationale to the patients and working with them in a collaborative manner.

\section{PATIENT COMPREHENSION/MENTAL STATUS}

It is often assumed that all patients will comprehend assessment material and instructions. However, this is often not the case. The assessor needs to be aware of the educational and reading level of patients to be certain that the material presented can be comprehended. Relatedly, the clinician needs to be aware of the mental status of the patient. Is there any sign of patient disorientation or possible cognitive impairment? Obviously, such impairment may invalidate basic testing in general, perhaps prompting for a neuropsychological evaluation. Evaluators need to be aware of such possibilities.

\section{IATROGENIC EFFECTS}

An iatrogenic effect is defined as an unwanted effect or perception induced inadvertently by a health care professional or his/her assessment or treatment. Often, patients may be told that bed rest is the only appropriate means of reducing pain, and are encouraged not to exercise too soon. This results in patients' belief that they have a functional deficit that cannot be immediately dealt with in any reasonable manner. Likewise, sometimes when pain persists after surgery due to iatrogenic effects caused by the surgeon's indicating that the surgery may produce 
its own nerve damage or other anatomic side effects. Therefore, the clinician must exercise care in talking with patients, because they will "carry away with them" information provided by the clinician which they believe is the "gospel truth," even though it may not be accurate. It is, therefore, important for an evaluator to become aware of what has been told to patients by other health care professionals in order to ascertain whether patients are presented with inaccurate beliefs that may affect their evaluation.

\section{SUMMARY AND CONCLUSIONS}

Some of the major psychosocial barriers to assessment/recovery that have been implicated as influencing the self-assessment of function have been reviewed. Secondary gain issues, such as financial gains, have traditionally been viewed as major barriers to recovery, especially in patients with workers' compensation injuries. However, this is an overly simplistic view because research has demonstrated that, even though exaggerated self-reports of physical symptoms are sometimes more extreme when financial secondary gain exists, favorable resolutions of such claims do not necessarily resolve the perceived pain or disability. Techniques for managing secondary gain issues when they may be present were reviewed. As was also discussed, an equally large potential barrier to assessment and treatment may revolve around secondary loss issues, such as financial, relationship loss and emotional consequences of loss. Health care professionals need to be aware of such losses because they are often associated with significant emotional distress. Emotional distress, in turn, may significantly affect the assessment process. A number of these distress factors were reviewed, such as depression, anxiety, and anger.

The presence of some major types of psychopathology can also affect the assessment process. In addition, somatization and symptom magnification issues were reviewed, as were 
compliance/resistance issues, patient comprehension/mental status, as well as iatrogenic effects. Thus, all of these potential barriers need to be kept in mind whenever evaluating a construct such as pain or function. One cannot assume that there will be one "gold standard" measure which will be the most reliable or valid. Rather, it was suggested that a multidimensional approach be used in such evaluations. This will provide potentially greater reliability in the self-assessment of function. 


\section{REFERENCES}

1. Gatchel RJ, How practitioners should evaluate personality to help manage patients with chronic pain, in Personality Characteristics of Patients with Pain, Gatchel RJ and Weisberg JN, Editors. 2000, American Psychological Association: Washington, DC.

2. Turk DC and Monarch ES, Biopsychosocial perspective on chronic pain, in Psychological Approaches to Pain Management: A Practitioner's Handbook, Turk DC and Gatchel RJ, Editors. 2002, Guilford: New York.

3. Flores L, Gatchel RJ, and Polatin PB, Objectification of functional improvement after nonoperative care. Spine, 1997. 22(14): p. 1622-33.

4. Gatchel RJ, A Compendium of Outcome Instruments for Assessment and Research of Spinal Disorders. 2001, LaGrange, IL: North American Spine Society.

5. Freud S, Introductory lectures on psychoanalysis, 1917. Vol. 16. 1959, London: Hogarth Press.

6. Leeman G, Polatin P, Gatchel R, and Kishino N, Managing secondary gain in patients with pain-associated disability: A clinical perspective. Journal of Workers Compensation, 2000.

7. Fishbain DA, Secondary gain concept: Definition problems and its abuse in medical practice. APS Journal, 1994. 3(4): p. 264-273.

8. King S, Concept of secondary gain--how valid is it? APS Journal, 1994. 3(4): p. 279-281.

9. Hammonds W, Brena S, and Unikel I, Compensation for work-related injuries and rehabilitation of patients with chronic pain. Southern Medical Journal, 1978. 71(6): p. 664-666. 
10. Rainville J, Sobel J, Hartigan C, and Wright A, The effect of compensation involvement of the reporting of pain and disability by patients referred for rehabilitation of chronic low back pain. Spine, 1997. 22(17): p. 2016-2024.

11. Evans R, Some observations on whiplash injuries. Neurologic Clinics, 1992. 10(4): p. 975-997.

12. Anderson T, Cole T, Gullickson G, Hudgens A, and Roberts A, Behavioral modification of chronic pain: A treatment program by a multidisciplinary team. Clinical Orthopedics and Related Research, 1977. 129: p. 96-100.

13. Schofferman J and Wasserman S, Successful treatment of low back pain and neck pain after a motor vehicle accident despite litigation. SPINE, 1994. 19(9): p. 1007-1010.

14. Gallagher R, Secondary gain in pain medicine--let us stick with biobehavioral data. APS Journal, 1994. 3(4): p. 274-278.

15. Dworkin R, Handlin D, Richlin D, Brand L, and Vannucci C, Unraveling the effects of compensation, litigation, and employment on treatment response in chronic pain. Pain, 1985. 23: p. 49-59.

16. Greenwood J, Intervention in work related disability: The need for an integrated approach. Social Science Medicine, 1984. 19: p. 595-601.

17. Romano JM and Turner JA, Chronic pain and depression: Does the evidence support a relationship? Psychological Bulletin, 1985. 97: p. 18-34.

18. Vlaeyen JWS, Kole-Snijders, Rooteveel A, Rusesink R, and Heuts P, The role of fear of movement/(re)injury in pain disability. Journal of Occupational Rehabilitation, 1995. 5: p. $235-252$. 
19. Fernandez E and Turk DC, The scope and significance of anger in the experience of chronic pain. Pain, 1995. 61: p. 165-175.

20. Kerns RD, Rosenberg R, and Jacob MC, Anger expression and chronic pain. Journal of Behavioral Medicine, 1994. 17: p. 57-68.

21. American Psychiatric Association, Diagnostic and Statistical Manual of Mental Disorders. 4th ed. 1994, Washington: APA. 
Table 1. Assumption Traps to Avoid When Considering the Best Outcome Measure to Use

- One cannot assume high correlations among different assessment measures.

- One cannot automatically assume, on an a priori basis, that one outcome measure will necessarily be more valid or reliable than another. Generally, the more objectively quantified the measure is, the more likely it can be empirically established as a valid and reliable referent or marker.

- One cannot assume that a physical measure will always be more objective than selfreport/psychosocial measures. No matter what the level of accuracy or sophistication of a mechanical device/physical assessment method used in collecting physiological measures, it is always the case that human interpretation ultimately must be used in the understanding of the resulting findings. Moreover, it must be remembered that a patient's performance during a physical assessment protocol can be greatly influenced by fear of pain or injury, motivation, instructional set, etc.

- One cannot assume that there is a consistent "pain prone" personality type that will negatively affect reliable and valid measurement. Research has consistently demonstrated no conclusive evidence for such a pain prone personality type.

- One should not assume that we are able to predict function with $100 \%$ accuracy. There are a great many individual differences (such as the psychosocial issues of motivation, fear of reinjury, etc.) that can significantly affect performance on a functional task. 
Table 2. Psychosocial Factors That Can Influence Self-Assessment of Function Secondary Gain Issues

\author{
Secondary Loss Issues
}

\author{
Emotional Distress
}

Psychopathology

Somatization and Symptom Magnification

Compliance and Resistance Issues

\author{
Patient Comprehension/Mental Status
}

Iatrogenic Effects 